\title{
普通小麦氮素利用效率相关性状全基因组关联分析
}

\author{
靳义荣 ${ }^{1, * *}$ 刘金栋 ${ }^{2, * *}$ 刘彩云 ${ }^{1} \quad$ 贾德新 $^{1} \quad$ 刘 鸠 $^{1, *}$ 王雅美 ${ }^{2, *}$
}

1 德州市农业科学研究院, 山东德州 $253015 ;^{2}$ 中国农业科学院深圳农业基因组研究所, 广东深圳 518124

摘 要: 氮元素在粮食作物生长和发育过程起着不可替代的作用。发掘氮素利用效率相关基因对于提升小麦产量、 减少环境污染具有重要意义。植株根系构型(root system architecture, RSA)代表着根系的结构及空间造型, 显著受氮 素水平影响。本研究在正常供氮和缺氮两种氮素水平下, 对 160 份来自黄淮冬麦区和北部冬麦区普通小麦品系的根 系构型相关性状 (总根长、总根表面积、总根体积、平均根直径和根尖数)进行统计, 并结合小麦 660K SNP (single nucleotide polymorphism)芯片基因分型数据对根系相关性状的相对值进行全基因组关联分析, 以期发掘氮素利用效 率相关位点。本研究检测到 34 个与氮素利用效率显著关联的 SNP 位点, 可解释 $6.9 \%$ 15.4\%的表型变异。关联位点 在所有染色体均有分布, 主要集中于 $1 \mathrm{~A} 、 2 \mathrm{~B} 、 3 \mathrm{~B} 、 5 \mathrm{~B} 、 6 \mathrm{~A} 、 6 \mathrm{~B}$ 和 $7 \mathrm{~A}$ 染色体。11 个位点与已报道位点重叠或接近, 其他 23 个位点可能为新的位点。另外, 在 $3 \mathrm{~B}$ 染色体上发现一个编码 E3 泛素连接酶的候选基因。

关键词：普通小麦; 根系构型; 氮素利用效率; 全基因组关联分析; 660K SNP 芯片

\section{Genome-wide association study of nitrogen use efficiency related traits in common wheat (Triticum aestivum L.)}

\author{
JIN Yi-Rong ${ }^{1, * *}$, LIU Jin-Dong ${ }^{2, * *}$, LIU Cai-Yun ${ }^{1}$, JIA De-Xin $^{1}$, LIU Peng ${ }^{1, *}$, and WANG Ya-Mei ${ }^{2, *}$ \\ ${ }^{1}$ Dezhou Academy of Agricultural Sciences, Dezhou 253015, Shandong, China; ${ }^{2}$ Agricultural Genomics Institute at Shenzhen, Chinese Academy of \\ Agricultural Sciences, Shenzhen 518124, Guangdong, China
}

\begin{abstract}
Nitrogen application plays an important role in plant growth and development. Exploring genetic loci related to nitrogen use efficiency is of great significance for improving wheat yield and reducing environmental pollution. Root system architecture (RSA) determined the composition of plant root system, and significantly affected by nitrogen level. Under different nitrogen levels (deficiency and normal), 160 winter wheat accessions from the Huanghuai valley and Northern winter wheat region were counted for their root architecture-related traits (total root length, total root surface area, total root volume, average root diameter, and root tip number). Genotype was analyzed using 660K SNP (single nucleotide polymorphism) data. Genome-wide association study (GWAS) was employed to identify the relevant loci for nitrogen use efficiency. A total of 34 associated loci were detected, which explained $6.9 \%-15.4 \%$ of the phenotypic variation. These loci distributed on all chromosomes and mainly centered on chromosomes 1A, 2B, 3B, 5B, 6A, 6B, and 7A, respectively. Among the loci detected in this study, 11 loci overlapped or were close to the reported ones, while the other 23 might be novel loci. In addition, we explored a candidate gene encoding the E3 ubiquitin ligase. This study is of great significance for understanding the genetic mechanism of nitrogen utilization and breeding high-yield wheat varieties.
\end{abstract}

Keywords: common wheat; root architecture; nitrogen use efficiency; GWAS; 660K SNP chip

\footnotetext{
本研究由山东省西部经济隆起带基层科技人才支持计划项目(XB2018FW029), 山东省重点研发计划项目(2017GNC10107), 山东省自 然科学基金项目(ZR2017BC015)和山东省农业科学院农业科技创新工程项目(CXGC2016B01)资助。

This study was supported by the Support Project for Grassroots Scientific and Technological Talents of Shandong Western Economic Swells up Belt (XB2018FW029), the Key Research and Development Program of Shandong Province (2017GNC10107), the Natural Science Foundation of Shandong Province (ZR2017BC015), and the Agricultural Science and Technology Innovation Project of Shandong Academy of Agricultural Sciences (CXGC2016B01).

*通信作者(Corresponding authors): 刘鹏, E-mail: liup9@163.com; 王雅美, E-mail: wangyamei@caas.cn

** 同等贡献(Contributed equally to this work)

第一作者联系方式：靳义荣, E-mail: jyr2014@163.com; 刘金栋, E-mail: liujindong_1990@163.com
}

Received (收稿日期): 2020-03-24; Accepted (接受日期): 2020-09-13; Published online (网络出版日期): 2020-10-15.

URL: https://kns.cnki.net/kcms/detail/11.1809.S.20201015.1022.002.html 
氮元素在植物正常生长发育过程中发挥着重要 作用。但是, 长期以来, 小麦生产过程中氮肥施用量 严重超出实际需求量，氮肥利用率只有 $21.2 \%$ $35.9 \%$ 。大量损失的氮素进入大气和地下水中，严重 污染水环境。另一方面, 贫困地区田间氮肥施用水 平较低, 小麦产量长期处于较低水平。开展小麦氮 素利用效率, 选育氮高效的新品种是提高产量和品 质，降低环境危害的有效途径。

根系作为植物最为重要的地下器官, 在植株生 长发育过程中发挥着重要作用 ${ }^{[1-3]}$ 。根系构型(root system architecture, RSA)主要指根系的结构及空间 造型, 包括多个相关性状, 例如总根长、总根表面 积、总根体积、根尖数和平均根直径等 ${ }^{[3-4]}$, 是影响 作物产量、品质和抗性最为重要的因素之一, 与氮 素吸收和转运等生理特性密切相关 ${ }^{[5-7]}$ 。植株根系 长度, 表面积和体积决定着植株氮素的吸收利用效 率 ${ }^{[3]}$ 。拥有大直径木质部导管的粗根系更利于穿透 深层次土壤, 提高养分吸收效率 ${ }^{[3]}$ 。另外, 根尖数 增加可有效提升根表面积和体积, 影响养分利用效 率 ${ }^{[6,8]}$ 。根系的根长、直径和吸收面积等性状与千粒 重、粒长、粒宽等产量性状呈显著正相关 ${ }^{[3]}$ 。因此，根 系构型是植物氮素利用能力的体现 ${ }^{[6,8]}$ 。

氮素利用效率是典型的复杂数量性状，由多基 因共同控制。QTL 定位(quantitative trait locus)又称 连锁分析, 是研究数量性状遗传功能的常用方法。 在大田条件下定位氮素利用效率相关性状的 QTL 位 点, 具有试验周期长, 且易受环境条件影响的缺点。 相较于传统的连锁分析, 全基因组关联分析 (genome-wide association study, GWAS)以自然群体 为材料, 来源广泛 (野生种、地方品种、现代品种和 高代品系), 基因多态性较高, 无需构建双亲群体, 具有发掘效率高、分辨率高且成本较低的优点。近 年来, 小麦 SNP 技术快速发展, 国内外已开发出多 款小麦 SNP 芯片, 如 9K、90K、660K 和 $820 \mathrm{~K}$ 芯片, 为 GWAS 分析提供了极为丰富且覆盖度较高的基因 型数据。迄今为止, 基于 SNP 标记的 GWAS 分析在 小麦加工品质 ${ }^{[9-10]}$ 、生理 ${ }^{[11-12]}$ 和抗病性 ${ }^{[9,13-14]}$ 等相关 性状基因的发掘研究中已广泛使用。

植物中拥有复杂的氮响应机制, 在不同的氮素 水平条件下会引起植物形成一系列的生理和生化变 化。 $\mathrm{NH}_{4}{ }^{+}$可通过 IAA、GA 或乙烯等方式生成信号 作用于下游调控蛋白以调节植物根系生长 ${ }^{[1-2]}$ 。苗期 根部性状作为表型进行氮素利用效率位点挖掘已在
相关研究中广泛应用。Lian 等 ${ }^{[15]}$ 在差异氮素含量水 平下对水稻苗期根系组成性状开展 QTL 定位, 发现 仅个别 QTL 在多个环境下共同存在, 认为不同氮素 环境下根系相关性状的定位可用于解析植物氮素利 用效率遗传机制。An 等 ${ }^{[16]}$ 以小麦为研究材料, 在大 田试验条件下分别对氮素吸收效率和苗期根部性状 进行遗传定位, 发现多个苗期根系发育相关性状的 QTL 与田间氮吸收的 QTL 位点一致。以上研究表明, 对植株根系生长发育相关性状进行探究, 可有效反 映植株氮素利用效率的遗传特征, 解析氮素利用效 率遗传机制。通过大田或苗期水培实验, 在不同的 氮素水平下已发掘一批影响小麦植株根系、植株生 长以及产量相关性状的 QTL 位点, 主要集中于小麦 $2 \mathrm{~A} 、 2 \mathrm{~B} 、 4 \mathrm{~A} 、 5 \mathrm{~A} 、 7 \mathrm{~A}$ 和 7B 染色体上 ${ }^{[16-19]}$ 。

我国黄淮冬麦区和北部冬麦区是小麦最为重要 的产区, 是保障我国粮食安全的重要基础。本研究 以 160 份来自于黄淮和北部冬麦区的育成品种、高 代品系和地方品种为材料, 在正常供氮和缺氮两种 氮素水培环境下研究不同基因型苗期根系构型相关 性状, 并结合小麦 $660 \mathrm{~K}$ 芯片进行 GWAS 分析, 以发 掘氮元素利用效率相关基因, 为培育高氮素利用效 率小麦品种提供参考。

\section{1 材料与方法}

\section{1 试验材料}

采用 160 份主要来自于黄淮和北部冬麦区的小 麦地方品种(20 份)、育成品种(76 份)和高代育种品 系(64 份)在不同氮素水平水培条件下进行氮素利用 效率相关性状的 GWAS 分析(附表 1)。

\section{2 水培试验}

试验在室内水培条件下进行。采用两种营养液: 完全营养液和缺氮营养液。营养液配制参考 Hoagland 营养液 ${ }^{[20]}$, 根据小麦苗期生长需求略有改 动。完全营养液组分如下: $1.8 \mathrm{mmol} \mathrm{L} \mathrm{L}^{-1} \mathrm{KCl} 、 1.5$ $\mathrm{mmol} \mathrm{L}^{-1} \mathrm{CaCl}_{2} 、 1.0 \mathrm{mmol} \mathrm{L}^{-1} \mathrm{Ca}\left(\mathrm{NO}_{3}\right)_{2} 、 1.0 \mathrm{mmol} \mathrm{L}^{-1}$ $\left(\mathrm{NH}_{4}\right)_{2} \mathrm{SO}_{4} 、 0.5 \mathrm{mmol} \mathrm{L} \mathrm{L}^{-1} \mathrm{MgSO}_{4} 、 0.2 \mathrm{mmol} \mathrm{L}^{-1}$ $\mathrm{KH}_{2} \mathrm{PO}_{4} 、 1.0 \times 10^{-3} \mathrm{mmol} \mathrm{L}^{-1} \mathrm{H}_{3} \mathrm{BO}_{3} 、 1.0 \times 10^{-3} \mathrm{mmol} \mathrm{L}^{-1}$ $\mathrm{ZnSO}_{4} 、 1.0 \times 10^{-3} \mathrm{mmol} \mathrm{L}^{-1} \mathrm{MnSO}_{4} 、 0.5 \times 10^{-3} \mathrm{mmol} \mathrm{L}^{-1}$ $\mathrm{CuSO}_{4} 、 0.1 \mathrm{mmol} \mathrm{L}{ }^{-1} \mathrm{Fe}-\mathrm{EDTA} 、 1.0 \times 10^{-4} \mathrm{mmol} \mathrm{L}^{-1}$ $\left(\mathrm{NH}_{4}\right)_{6} \mathrm{Mo}_{7} \mathrm{O}_{24}$ 。缺氮营养液的组分如下: $1.8 \mathrm{mmol} \mathrm{L}^{-1}$ $\mathrm{KCl} 、 1.5 \mathrm{mmol} \mathrm{L}^{-1} \mathrm{CaCl}_{2} 、 1.0 \mathrm{mmol} \mathrm{L}{ }^{-1} \mathrm{Ca}\left(\mathrm{NO}_{3}\right)_{2} 、 0.5$ mmol L ${ }^{-1} \mathrm{MgSO}_{4} 、 0.2 \mathrm{mmol} \mathrm{L}{ }^{-1} \mathrm{KH}_{2} \mathrm{PO}_{4} 、 1.0 \times 10^{-3}$ $\mathrm{mmol} \mathrm{L}^{-1} \mathrm{H}_{3} \mathrm{BO}_{3} 、 1.0 \times 10^{-3} \mathrm{mmol} \mathrm{L}^{-1} \mathrm{ZnSO}_{4} 、 1.0 \times 10^{-3}$ 
$\mathrm{mmol} \mathrm{L}^{-1} \mathrm{MnSO}_{4} 、 0.5 \times 10^{-3} \mathrm{mmol} \mathrm{L}^{-1} \mathrm{CuSO}_{4}, 0.1 \mathrm{mmol}$ $\mathrm{L}^{-1} \mathrm{Fe}$-EDTA、 $1.0 \times 10^{-4} \mathrm{mmol} \mathrm{L}^{-1}\left(\mathrm{NH}_{4}\right)_{6} \mathrm{Mo}_{7} \mathrm{O}_{24}$ 。

挑选饱满、大小一致的小麦籽粒置于 $10 \% \mathrm{H}_{2} \mathrm{O}_{2}$ 溶液中浸泡 $10 \mathrm{~min}$, 去离子水冲洗 3 次后, 置于湿润 滤纸上培养。在一叶一心期, 选取长势一致的幼苗, 固定在育苗盘中, 将育苗盘分别置于完全营养液和 缺氮营养液中。通过氧气原对营养液进行持续通气, 并且每隔 $3 \mathrm{~d}$ 更换一次营养液, 培养至 $21 \mathrm{~d}$ 后, 用去 离子水将根部冲洗干净, 用剪刀将根和茎在分节处 剪断, 分别置于自封袋中备测。

\section{3 根系相关表型测定}

于每份样品中随机选择 10 个单株, 将各单株侧 根逐一分开, 在不重叠的状态下置于装有去离子水 的透明根盘中, 利用 WinRHIZOLA6400XL 透视扫 描系统对根系进行扫描, 获取各单株根系图片。然 后利用 WinRHIZOPro 软件分析各样品的根尖数 (number of root tips, NRT)、根系总长度(total root length, TRL)、根系总表面积(total root surface area, TRS)、根系总体积(total root volume, TRV)和根系平 均直径(average root diameter, ARD)等表型数据, 取 3 次重复的平均值作为材料的表型数据值。随后, 计 算 2 种不同氮素条件下各性状的相对值, 即相对根 尖数(relative number of root tips, RNRT)、相对总根 长(relative total root length, RTRL)、相对总根表面积 (relative total root surface area, RTRS)、相对总根体积 (relative total root volume, RTRV)和相对根平均直径 (relative average root diameter, RARD)。采用 SAS 9.2 (www.sas.com)软件统计分析数据, 并计算相关系数。

\section{4 基因型检测}

利用 CTAB 法提取小麦幼嫩叶片基因组 $\mathrm{DNA}^{[21]}$ 。本研究基因型分析采用小麦 $660 \mathrm{~K}$ SNP 芯 片 (包含 630,517 个 SNP 标记) (博奥晶典, http:// www.capitalbiotech.com/) 在剔除缺失染色体定位信 息和物理位置的标记后, 篮选最小等位基因频率 (minor allele frequency, MAF) > 0.05 和缺失率 (missing rate) $<20 \%$ (PowerMarker v3.25)的标记用 于下一步 GWAS 分析。利用多态性信息含量 (polymorphism information content, PIC)和基因多态 性两个参数对黄淮冬麦区和北部冬麦区群体遗传多 态性进行分析。PIC 值基于 PowerMarker v3.25 按照 以下公式计算: $\mathrm{PIC}=1-\sum\left(p_{i}\right)^{2}$ 。其中, $p_{i}$ 为第 $i$ 个等 位基因在群体中出现的频率。基因多态性的计算也 由 PowerMarker v3.25 完成。

\section{5 主成分分析 (principal components analysis,} PCA)和 LD 衰减分析

PCA 分析通过 Tassel v5.0 (https://www.maizegenetics.net/tassel)开展。PCA 可视化在 R 环境下通 过 Pot3D (http://www.harrisgeospatial.com/docs/ PLOT3D. html) 和 Satterplot $3 d$ (http://www.sthda.com/english/ wiki/scatterplot3d-3d-graphics-r-software-and-datavisualization)程序包完成。利用 Tassel v5.0 计算标记 间 LD。

\section{6 全基因组关联分析}

应用 Tassel v5.0软件中的 MLM 模型，以主成分 分析 (PCA) 和亲缘关系 (Kinship) 作为协变量, 结 合篮选后的基因型数据, 对 160 份小麦品系根系构 型相关性状进行关联分析。GWAS 分析结果中, 当 $P \leq 0.001$ 时, 认为该标记与性状显著关联。曼哈顿 图和 Q-Q 图可视化在 R 环境中通过 CMplot 程序包 (https://github.com/YinLiLin/R-CMplot)完成。

\section{2 结果与分析}

\section{1 表型数据分析}

160 份普通小麦材料在完全营养液中 NRT、TRL、 TRS、TRV 和 ARD 的均值分别为 $119.97 、 210.23 \mathrm{~cm}$ 、 $19.62 \mathrm{~cm}^{2} 、 0.42 \mathrm{~cm}^{3}$ 和 $0.46 \mathrm{~mm}$; 在缺氮营养液中 NRT、TRL、TRS、TRV 和 ARD 的均值分别为 476.83、 $406.85 \mathrm{~cm} 、 22.97 \mathrm{~cm}^{2} 、 0.62 \mathrm{~cm}^{3}$ 和 $0.62 \mathrm{~mm}$ (表 1, 附 表 1, 附图 1, 附图 2, 附图 3)。160 份材料在 2 种营 养液中 RNRT、RTRL、RTRS、RTRV 和 RARD 的均 值分别为: $5.15(0.59 \sim 16.60) 、 2.10(0.92 \sim 5.83) 、 1.21$ (0.74 2.27)、1.49 (0.95 2.52) 和 $1.44(0.53 \sim 3.82)$ 。2 种培养环境下根系相关性状比值均呈连续性分布, 变异范围广, 遗传基础丰富, 适于进行 GWAS 分析 (附图 4)。对各性状开展相关性分析, 2 种培养条件下 根系构成相关性状比值呈显著正相关。其中, RTRL 与 RTRS $(r=0.718) 、 \operatorname{RARD}(r=0.383) 、 \operatorname{RTRV}(r=$ $0.484) 、 \operatorname{RNRT}(r=0.513)$ 呈显著正相关 $(P<0.05)$; RTRS 与 RARD $(r=0.531) 、 \operatorname{RTRV}(r=0.475)$ 和 RNRT $(r=0.492)$ 呈显著正相关 $(P<0.05)$; RARD与 RNRT 呈显著正相关 $(r=0.514, P<0.05)$; RTRV 和 RNRT 也呈显著正相关 $(r=0.439, P<0.05)$ 。

\section{2 基因型分析}

为保证关联结果的准确性, 过滤掉物理位置缺 失、 $\mathrm{MAF}<5 \%$ 且缺失率 $>20 \%$ 的 SNP 标记, 最终 剩余 223,168 个标记用于进一步分析。标记覆盖所 
表 1160 份普通小麦品种根系构型相关性状表型统计

Table 1 Summary of root system architecture (RSA) traits under different nitrogen level in 160 common wheat accessions

\begin{tabular}{|c|c|c|c|c|c|c|}
\hline $\begin{array}{c}\text { 处理 } \\
\text { Treatment }\end{array}$ & $\begin{array}{l}\text { 性状 } \\
\text { Trait }\end{array}$ & $\begin{array}{c}\text { 极小值 } \\
\text { Min. }\end{array}$ & $\begin{array}{c}\text { 极大值 } \\
\text { Max. }\end{array}$ & $\begin{array}{c}\text { 均值 } \\
\text { Average }\end{array}$ & $\begin{array}{c}\text { 标准差 } \\
\text { Standard deviation }\end{array}$ & $\begin{array}{c}\text { 变异系数 } \\
\mathrm{CV}(\%)\end{array}$ \\
\hline 完全营养液 & TRL (cm) & 86.33 & 495.67 & 210.23 & 64.44 & 30.7 \\
\hline \multirow{4}{*}{$\begin{array}{l}\text { Absolute nutrient } \\
\text { solution }\end{array}$} & TRS $\left(\mathrm{cm}^{2}\right)$ & 11.25 & 26.85 & 19.62 & 3.48 & 17.7 \\
\hline & $\operatorname{TRV}\left(\mathrm{cm}^{3}\right)$ & 0.40 & 0.98 & 0.42 & 0.12 & 18.8 \\
\hline & $\mathrm{ARD}(\mathrm{mm})$ & 0.23 & 0.75 & 0.46 & 0.12 & 26.2 \\
\hline & NRT & 24.50 & 396.50 & 119.97 & 65.50 & 54.6 \\
\hline 缺氮营养液 & TRL (cm) & 189.52 & 631.29 & 406.85 & 94.21 & 23.2 \\
\hline N-deficiency & TRS $\left(\mathrm{cm}^{2}\right)$ & 14.62 & 26.87 & 22.97 & 2.27 & 9.9 \\
\hline \multirow[t]{3}{*}{ nutrient solution } & $\operatorname{TRV}\left(\mathrm{cm}^{3}\right)$ & 0.36 & 0.54 & 0.62 & 0.03 & 7.1 \\
\hline & $\mathrm{ARD}(\mathrm{mm})$ & 0.31 & 1.07 & 0.62 & 0.14 & 22.6 \\
\hline & NRT & 208.00 & 881.00 & 476.83 & 145.20 & 30.5 \\
\hline 相对比值 & RTRL & 0.92 & 5.83 & 2.10 & 0.75 & 35.7 \\
\hline \multirow[t]{4}{*}{ Relative ratio } & RTRS & 0.74 & 2.27 & 1.21 & 0.26 & 21.2 \\
\hline & RTRV & 0.95 & 2.52 & 1.49 & 0.28 & 19.2 \\
\hline & RARD & 0.53 & 3.82 & 1.44 & 0.50 & 34.6 \\
\hline & RNRT & 0.59 & 16.60 & 5.15 & 3.06 & 54.7 \\
\hline
\end{tabular}

TRL: 根系总长度; TRS: 根系总表面积; TRV: 根系总体积; ARD: 根系平均直径; NRT: 根尖数; RTRL: 相对总根长; RTRS: 相对总 根表面积; RTRV：相对总根体积; RARD：相对根平均直径; RNRT：相对根尖数。

TRL: total root length; TRS: total root surface area; TRV: total root volume; ARD: average root diameter; NRT: number of root tips; RTRL: relative total root length; RTRS: relative total root surface area; RTRV: relative total root volume; RARD: relative average root diameter; RNRT: relative number of root tips.

有染色体区段, 物理图谱总长度 $14,063.9 \mathrm{Mb}$, 平均 密度为 $0.097 \mathrm{Mb}$ /标记。 $\mathrm{A} 、 \mathrm{~B}$ 和 $\mathrm{D}$ 基因组长度分别 为 $4934.5 、 5179.0$ 和 $3950.4 \mathrm{Mb}$, 分别包含 93,664 (41.9\%)、108,936 (56.3\%)和 20,568 (9.2\%)个标记。B 基因组密度最高 $(0.048 \mathrm{Mb}$ 标记)，其次为 $\mathrm{A}$ 基因组 (0.052 Mb/标记), D 基因组标记密度最低 $(0.193 \mathrm{Mb} /$ 标记，表 2)。

\subsection{PCA 分析和 LD 衰减分析}

利用 Tassel v 5.0160 份小麦品系进行 PCA 分析, 发现 160 份小麦品系可分为 2 个亚群(图 1)。其中, 亚 群 1 包含 20 份农家种, 亚群 2 包含 140 份育成品种 和高代品系。以第 95 百分位的 $r^{2}$ 值作为阈值估测 $\mathrm{LD}$ 衰减距离, 160 份小麦品系全基因组水平的 $r^{2}$ 阈 值分别为 0.135 。 $r^{2}$ 國值与 LD 衰减曲线的交叉点为 $\mathrm{LD}$ 衰减距离 ${ }^{[22]}$ 。本研究中, 全基因组平均 LD 衰减 距离为 $8 \mathrm{Mb}$ (图 2)。

\section{4 根系构型相关性状 GWAS 分析}

GWAS 分析中出现假阳性结果的最主要原因是 材料背景复杂, 存在群体结构现象 ${ }^{[23]}$ 。PCA 分析表 明前 2 个主成分分别可解释表型变异的 $35.4 \%$ 和 $8.6 \%, 160$ 份小麦品系可分为 2 个亚群，存在显著的 群体结构。结合群体结构 (PCA)和亲缘关系 $(\mathrm{K})$ 的混 合线性模型(MLM)可有效降低由群体结构造成的假

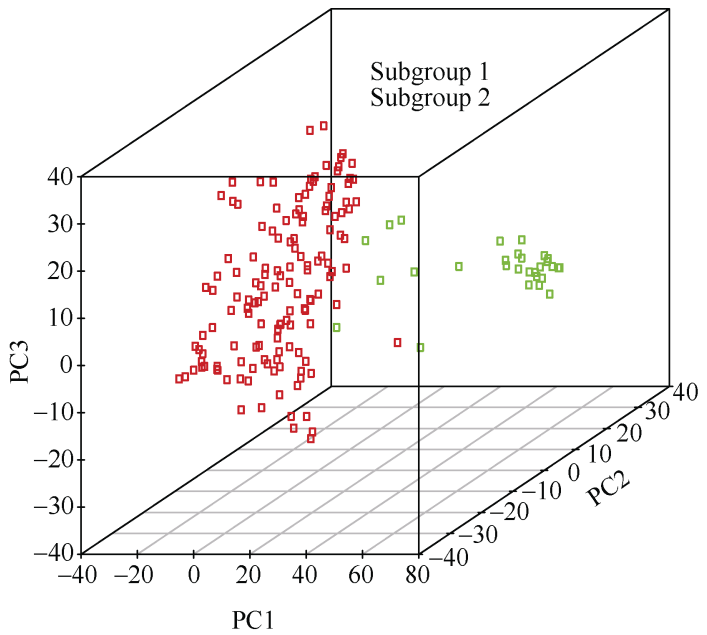

图 1160 份小麦品系主成分分析

Fig. 1 Principal component analysis of 160 common wheat lines

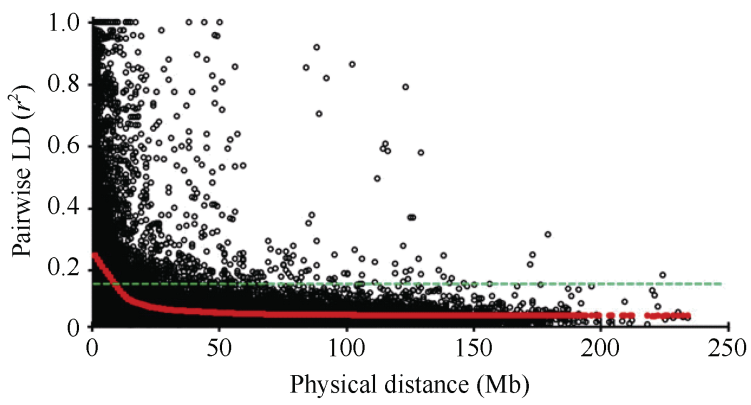

图 2160 份小麦品系 LD 衰减分析

Fig. 2 LD decay analysis of 160 common wheat lines 
表 2160 份普通小麦品系基因型数据统计

Table 2 Genotype data statistics of 160 common wheat lines

\begin{tabular}{|c|c|c|c|c|c|c|c|c|c|}
\hline \multirow{2}{*}{$\begin{array}{c}\text { 染色体 } \\
\text { Chr. }\end{array}$} & \multirow{2}{*}{$\begin{array}{l}\text { 标记数目 } \\
\text { Number of } \\
\text { markers }\end{array}$} & \multirow{2}{*}{$\begin{array}{l}\text { 长度 }{ }^{a} \\
\text { Length }^{\mathrm{a}} \\
(\mathrm{Mb})\end{array}$} & \multirow{2}{*}{$\begin{array}{c}\text { 密度 } \\
\text { Density } \\
(\mathrm{Mb} / \text { marker) }\end{array}$} & \multicolumn{2}{|c|}{$\begin{array}{c}\text { 最小等位基因频率 } \\
\text { Minimum allele frequency } \\
\text { (MAF) }\end{array}$} & \multicolumn{2}{|c|}{$\begin{array}{c}\text { 基因多态性 } \\
\text { Genetic } \\
\text { diversity }\end{array}$} & \multicolumn{2}{|c|}{$\begin{array}{c}\text { 多态性信息含量 } \\
\text { Polymorphic information } \\
\text { content (PIC) }\end{array}$} \\
\hline & & & & $\begin{array}{c}\text { 均值 } \\
\text { Average }\end{array}$ & $\begin{array}{c}\text { 范围 } \\
\text { Range }\end{array}$ & $\begin{array}{c}\text { 均值 } \\
\text { Average }\end{array}$ & $\begin{array}{c}\text { 范围 } \\
\text { Range }\end{array}$ & $\begin{array}{c}\text { 均值 } \\
\text { Average }\end{array}$ & $\begin{array}{c}\text { 范围 } \\
\text { Range }\end{array}$ \\
\hline $1 \mathrm{~A}$ & 18,975 & 594.00 & 0.031 & 0.26 & $0.05-0.50$ & 0.37 & $0.10-0.50$ & 0.28 & $0.09-0.38$ \\
\hline $1 \mathrm{~B}$ & 13,810 & 689.40 & 0.050 & 0.31 & $0.05-0.50$ & 0.39 & $0.10-0.50$ & 0.29 & $0.09-0.38$ \\
\hline $1 \mathrm{D}$ & 4464 & 495.40 & 0.111 & 0.18 & $0.05-0.49$ & 0.34 & $0.10-0.50$ & 0.28 & $0.09-0.38$ \\
\hline $2 \mathrm{~A}$ & 2710 & 780.70 & 0.288 & 0.27 & $0.05-0.50$ & 0.36 & $0.10-0.50$ & 0.30 & $0.09-0.38$ \\
\hline $2 \mathrm{~B}$ & 14,287 & 801.20 & 0.056 & 0.25 & $0.05-0.50$ & 0.38 & $0.10-0.50$ & 0.30 & $0.09-0.38$ \\
\hline $2 \mathrm{D}$ & 3604 & 651.60 & 0.181 & 0.32 & $0.05-0.50$ & 0.36 & $0.10-0.50$ & 0.30 & $0.09-0.38$ \\
\hline $3 \mathrm{~A}$ & 13,177 & 750.80 & 0.057 & 0.26 & $0.05-0.50$ & 0.36 & $0.10-0.50$ & 0.27 & $0.09-0.38$ \\
\hline $3 \mathrm{~B}$ & 20,116 & 830.30 & 0.041 & 0.27 & $0.05-0.50$ & 0.40 & $0.10-0.50$ & 0.31 & $0.09-0.38$ \\
\hline $3 \mathrm{D}$ & 2417 & 615.40 & 0.255 & 0.20 & $0.05-0.50$ & 0.31 & $0.10-0.50$ & 0.26 & $0.09-0.38$ \\
\hline $4 \mathrm{~A}$ & 12,438 & 744.50 & 0.060 & 0.25 & $0.05-0.50$ & 0.38 & $0.10-0.50$ & 0.30 & $0.09-0.38$ \\
\hline 4B & 10,680 & 673.40 & 0.063 & 0.23 & $0.05-0.50$ & 0.36 & $0.10-0.50$ & 0.28 & $0.09-0.38$ \\
\hline $4 \mathrm{D}$ & 1055 & 509.60 & 0.483 & 0.26 & $0.05-0.49$ & 0.38 & $0.10-0.50$ & 0.31 & $0.09-0.38$ \\
\hline $5 \mathrm{~A}$ & 11,972 & 709.70 & 0.059 & 0.27 & $0.05-0.50$ & 0.37 & $0.10-0.50$ & 0.29 & $0.09-0.38$ \\
\hline $5 \mathrm{~B}$ & 23,584 & 713.00 & 0.030 & 0.29 & $0.05-0.50$ & 0.37 & $0.10-0.50$ & 0.29 & $0.09-0.38$ \\
\hline $5 \mathrm{D}$ & 2512 & 566.00 & 0.225 & 0.29 & $0.05-0.50$ & 0.33 & $0.10-0.50$ & 0.27 & $0.09-0.38$ \\
\hline $6 \mathrm{~A}$ & 14,250 & 618.00 & 0.043 & 0.25 & $0.05-0.50$ & 0.40 & $0.10-0.50$ & 0.32 & $0.09-0.38$ \\
\hline $6 \mathrm{~B}$ & 14,434 & 720.90 & 0.050 & 0.27 & $0.05-0.50$ & 0.33 & $0.10-0.50$ & 0.27 & $0.09-0.38$ \\
\hline $6 \mathrm{D}$ & 2544 & 473.50 & 0.186 & 0.25 & $0.05-0.50$ & 0.36 & $0.10-0.50$ & 0.27 & $0.09-0.38$ \\
\hline $7 \mathrm{~A}$ & 20,807 & 736.60 & 0.035 & 0.27 & $0.05-0.50$ & 0.32 & $0.10-0.50$ & 0.29 & $0.09-0.38$ \\
\hline $7 \mathrm{~B}$ & 12,025 & 750.60 & 0.062 & 0.28 & $0.05-0.50$ & 0.36 & $0.10-0.50$ & 0.28 & $0.09-0.38$ \\
\hline $7 \mathrm{D}$ & 4537 & 638.60 & 0.141 & 0.22 & $0.05-0.50$ & 0.29 & $0.10-0.50$ & 0.26 & $0.09-0.38$ \\
\hline A genome & 93,664 & 4934.50 & 0.052 & 0.27 & $0.05-0.50$ & 0.38 & $0.10-0.50$ & 0.29 & $0.09-0.38$ \\
\hline B genome & 108,936 & 5179.00 & 0.048 & 0.29 & $0.05-0.50$ & 0.36 & $0.10-0.50$ & 0.28 & $0.09-0.38$ \\
\hline D genome & 20,568 & 3950.40 & 0.193 & 0.24 & $0.05-0.50$ & 0.33 & $0.10-0.50$ & 0.28 & $0.09-0.38$ \\
\hline
\end{tabular}

a 标记物理位置来源于中国春小麦参考基因组(RefSeq v1.0, http://www.wheatgenome.org/; IWGSC, 2018)。

${ }^{a}$ physical positions of SNP markers were based on the Chinese Spring reference genome in IWGSC (RefSeq v1.0, http://www.wheatgenome.org/; IWGSC, 2018).

阳性现象 ${ }^{[24-25]}$ 。因此, 本研究中, 为了避免由于群体 结构和亲缘关系引起的假阳性，我们采用基于 Tassel v 5.0 的 MLM 模型对不同氮素条件下根系构 型相关性状比值进行 GWAS 分析。

基于两种不同氮素水平下根系构型相关性状的 比值共计检测到 86 个与氮素利用效率相关性状显 著关联的 SNP 标记, 分布于 34 个遗传位点(表 3 和 图 3)。其中, 7 个位点与 RNRT 相关，分布于 1B、2B、 $2 \mathrm{D} 、 3 \mathrm{~A} 、 3 \mathrm{~B}$ (2) 和 $4 \mathrm{~B}$ 染色体上, 分别解释 $8.4 \% \sim 13.8 \%$ 的表型变异; 2 个位点与 RARD 关联, 分 布于 $2 \mathrm{~B}$ 和 $3 \mathrm{~B}$ 染色体上, 分别解释 $9.2 \%$ 和 $10.4 \%$ 的 表型变异; 7 个位点与 RTRL 显著关联, 分布于 $1 \mathrm{~A}$ 、 $1 \mathrm{~B} 、 2 \mathrm{~B} 、 2 \mathrm{D} 、 3 \mathrm{~B}$ 和 $5 \mathrm{~B}$ 染色体上, 分别解释 $6.9 \% \sim 11.9 \%$ 的表型变异; 18 个位点与 RTRS 相关,
分布于 $1 \mathrm{~A} 、 1 \mathrm{~B} 、 2 \mathrm{~A} 、 3 \mathrm{~B} 、 4 \mathrm{~B} 、 5 \mathrm{~A} 、 5 \mathrm{~B} 、 6 \mathrm{~A} 、 6 \mathrm{~B} 、$ $6 \mathrm{D} 、 7 \mathrm{~A}$ 和 $7 \mathrm{~B}$ 染色体上, 分别解释 $9.2 \% \sim 14.8 \%$ 的表 型变异; 29 个位点与 RTRV 相关，分布于 $1 \mathrm{~A} 、 1 \mathrm{~B}$ 、 $1 \mathrm{D} 、 2 \mathrm{~A} 、 2 \mathrm{~B} 、 2 \mathrm{D} 、 3 \mathrm{~A} 、 3 \mathrm{~B} 、 4 \mathrm{~B} 、 5 \mathrm{~A} 、 5 \mathrm{~B} 、 6 \mathrm{~A} 、$ $6 B 、 7 A$ 和 $7 B$ 染色体上, 分别解释 $9.6 \%$ 19.6\%的表 型变异(表 3 和图 3)。不同氮素水平环境下根系构成 相关性状比值存在较高的相关性。相应的, 在关联 结果中我们发现多个相对性状有相同遗传位点, 如 11 个位点分别与 RTRV 和 RTRS 相关; 2 个位点与 RTRV、RTRS 和 RTRL 相关; 2 个位点与 RTRV 和 RTRL 相关; 1 个位点与 RNRT、RTRV 和 RTRL 相 关; 2 个位点与 RNRT 和 RTRV 相关; 2 个位点与 RNRT、RTRV 和 RTRS 相关; 1 个位点与 RTRV 和 RTRS 相关。 
表 3 与氮素利用效率显著关联 $(P \leq 0.001)$ 的 SNP 标记

Table 3 Marker-trait associations for nitrogen use efficiency related traits $(P \leq 0.001)$

\begin{tabular}{|c|c|c|c|c|c|c|c|}
\hline $\begin{array}{l}\text { 性状 } \\
\text { Trait }\end{array}$ & $\begin{array}{c}\text { 标记 } \\
\text { Marker }\end{array}$ & $\begin{array}{c}\text { 染色体 } \\
\text { Chromosome }\end{array}$ & $\begin{array}{c}\text { 位置 } \\
\text { Position }(\mathrm{Mb})\end{array}$ & $\begin{array}{c}\text { 优异等位基因 } \\
\text { Favorable allele }\end{array}$ & $\begin{array}{c}P \text { 值 } \\
P \text {-value }\end{array}$ & $\begin{array}{c}\text { 表型解释变异 } \\
R^{2}(\%)\end{array}$ & $\begin{array}{l}\text { 参考文献 } \\
\text { Reference }\end{array}$ \\
\hline RNRT & $A X \_110370425$ & $1 \mathrm{~B}$ & 577.6 & G & $5.38 \mathrm{E}-04$ & 9.7 & \\
\hline RNRT & $A X \_109497462$ & $2 \mathrm{~B}$ & 756.7 & $\mathrm{~T}$ & $9.75 \mathrm{E}-04$ & 8.9 & \\
\hline RNRT & $A X \_108811964$ & $2 \mathrm{D}$ & 612.3 & $\mathrm{C}$ & $4.81 \mathrm{E}-04$ & 10.0 & \\
\hline RNRT & $A X \_109933410$ & $3 \mathrm{~A}$ & 22.6 & G & $3.45 \mathrm{E}-04$ & 8.4 & \\
\hline RNRT & $A X \_109931146$ & $3 B$ & 117.6 & $\mathrm{~T}$ & $3.78 \mathrm{E}-04$ & 10.2 & Ren et al. ${ }^{[29]}$ \\
\hline RNRT & $A X \_110438119$ & $3 \mathrm{~B}$ & 778.3 & $\mathrm{~T}$ & $2.68 \mathrm{E}-04$ & 11.2 & \\
\hline RNRT & $A X \_109838302$ & $4 \mathrm{~B}$ & 540.3 & $\mathrm{~T}$ & $2.84 \mathrm{E}-05$ & 13.8 & \\
\hline RARD & $A X \_108964719$ & $2 \mathrm{~B}$ & 207.1 & G & $9.08 \mathrm{E}-04$ & 9.2 & \\
\hline RARD & $A X \_110968453$ & $3 \mathrm{~B}$ & 386.4 & G & $4.06 \mathrm{E}-04$ & 10.4 & \\
\hline RTRL & $A X \_108913559$ & $1 \mathrm{~A}$ & 515.8 & $\mathrm{C}$ & $9.88 \mathrm{E}-04$ & 7.0 & \\
\hline RTRL & $A X \_110617713$ & $1 \mathrm{~B}$ & 633.3 & $\mathrm{~T}$ & $6.67 \mathrm{E}-04$ & 9.6 & \\
\hline RTRL & $A X \_109031690$ & $2 \mathrm{~B}$ & 25.5 & $\mathrm{~T}$ & $6.79 \mathrm{E}-04$ & 9.6 & \\
\hline RTRL & $A X_{-} 108858890$ & $2 \mathrm{~B}$ & 26.0 & $\mathrm{~T}$ & $5.03 \mathrm{E}-04$ & 10.4 & \\
\hline RTRL & $A X \_109958492$ & $2 \mathrm{~B}$ & 26.0 & G & $7.36 \mathrm{E}-04$ & 9.4 & \\
\hline RTRL & $A X \_108989039$ & $2 \mathrm{~B}$ & 26.1 & G & $7.36 \mathrm{E}-04$ & 9.4 & \\
\hline RTRL & $A X \_110583038$ & $2 \mathrm{~B}$ & 26.1 & G & $8.66 \mathrm{E}-04$ & 9.2 & \\
\hline RTRL & $A X \_110536069$ & $2 \mathrm{~B}$ & 26.1 & G & 4.07E-04 & 10.2 & \\
\hline RTRL & $A X \_109855057$ & $2 \mathrm{~B}$ & 26.1 & A & $7.73 \mathrm{E}-04$ & 9.3 & \\
\hline RTRL & $A X \_108758132$ & $2 \mathrm{~B}$ & 26.1 & $\mathrm{C}$ & $8.12 \mathrm{E}-04$ & 9.6 & \\
\hline RTRL & $A X \_109974238$ & $2 \mathrm{~B}$ & 785.8 & $\mathrm{~T}$ & $9.99 \mathrm{E}-04$ & 9.5 & \\
\hline RTRL & $A X \_108811964$ & $2 \mathrm{D}$ & 612.3 & $\mathrm{C}$ & $1.26 \mathrm{E}-04$ & 11.9 & \\
\hline RTRL & $A X \_109892051$ & $3 \mathrm{~B}$ & 821.0 & $\mathrm{~T}$ & $6.39 \mathrm{E}-04$ & 10.3 & \\
\hline RTRL & $A X \_110045465$ & $5 \mathrm{~B}$ & 707.1 & $\mathrm{C}$ & $5.19 \mathrm{E}-05$ & 13.0 & \\
\hline RTRS & $A X \_110543784$ & $1 \mathrm{~A}$ & 496.9 & A & $3.82 \mathrm{E}-04$ & 10.9 & \\
\hline RTRS & $A X \_109973556$ & $1 \mathrm{~A}$ & 520.9 & $\mathrm{C}$ & $3.62 \mathrm{E}-04$ & 10.3 & \\
\hline RTRS & $A X \_110111289$ & $1 \mathrm{~B}$ & 384.3 & G & $3.24 \mathrm{E}-04$ & 10.4 & \\
\hline RTRS & $A X \_108774755$ & $2 \mathrm{~A}$ & 125.9 & G & $4.60 \mathrm{E}-04$ & 9.9 & \\
\hline RTRS & $A X \_110075829$ & $3 \mathrm{~B}$ & 250.6 & G & $3.91 \mathrm{E}-04$ & 10.2 & \\
\hline RTRS & $A X_{-} 110612422$ & $3 \mathrm{~B}$ & 403.5 & G & $2.72 \mathrm{E}-04$ & 10.6 & \\
\hline RTRS & $A X_{-} 110411187$ & 3B & 410.1 & G & $7.86 \mathrm{E}-04$ & 9.2 & \\
\hline RTRS & $A X \_110388860$ & $3 B$ & 466.1 & G & 4.02E-04 & 10.1 & \\
\hline RTRS & $A X \_108977570$ & $3 \mathrm{~B}$ & 780.4 & G & $3.87 \mathrm{E}-04$ & 10.2 & \\
\hline RTRS & $A X \_109915316$ & $4 \mathrm{~B}$ & 554.8 & G & $4.40 \mathrm{E}-04$ & 10.0 & \\
\hline RTRS & $A X \_110522770$ & $5 \mathrm{~A}$ & 8.2 & $\mathrm{~T}$ & $4.58 \mathrm{E}-04$ & 10.1 & Ren et al. ${ }^{[29]}$ \\
\hline RTRS & $A X \_110044622$ & $5 \mathrm{~B}$ & 59.2 & $\mathrm{~T}$ & $3.82 \mathrm{E}-04$ & 10.2 & \\
\hline RTRS & $A X_{-} 110366842$ & $5 \mathrm{~B}$ & 60.2 & G & $4.52 \mathrm{E}-04$ & 10.0 & \\
\hline RTRS & $A X \_109440517$ & $5 \mathrm{~B}$ & 504.0 & $\mathrm{~T}$ & $4.23 \mathrm{E}-04$ & 10.0 & \\
\hline RTRS & $A X_{-} 108813077$ & $5 \mathrm{~B}$ & 517.9 & $\mathrm{C}$ & $4.56 \mathrm{E}-04$ & 9.9 & \\
\hline RTRS & $A X \_110045465$ & $5 \mathrm{~B}$ & 707.1 & $\mathrm{C}$ & $1.34 \mathrm{E}-05$ & 14.8 & \\
\hline RTRS & $A X \_108740494$ & $6 \mathrm{~A}$ & 564.9 & G & 4.87E-04 & 9.9 & \\
\hline RTRS & $A X_{-} 109907271$ & $6 \mathrm{~B}$ & 54.4 & $\mathrm{~T}$ & $2.13 \mathrm{E}-04$ & 11.2 & Ren et al. ${ }^{[29]}$ \\
\hline RTRS & $A X \_110402455$ & $6 \mathrm{D}$ & 469.2 & G & $6.43 \mathrm{E}-04$ & 9.6 & \\
\hline RTRS & $A X \_110447828$ & $6 \mathrm{D}$ & 472.3 & $\mathrm{~T}$ & 4.04E-04 & 10.3 & \\
\hline RTRS & $A X \_110444174$ & $7 \mathrm{~A}$ & 722.6 & $\mathrm{~T}$ & $1.21 \mathrm{E}-04$ & 11.8 & \\
\hline
\end{tabular}


(续表 3)

\begin{tabular}{|c|c|c|c|c|c|c|c|}
\hline $\begin{array}{l}\text { 性状 } \\
\text { Trait }\end{array}$ & $\begin{array}{c}\text { 标记 } \\
\text { Marker }\end{array}$ & $\begin{array}{c}\text { 染色体 } \\
\text { Chromosome }\end{array}$ & $\begin{array}{c}\text { 位置 } \\
\text { Position }(\mathrm{Mb})\end{array}$ & $\begin{array}{c}\text { 优异等位基因 } \\
\text { Favorable allele }\end{array}$ & $\begin{array}{c}P \text { 值 } \\
P \text {-value }\end{array}$ & $\begin{array}{c}\text { 表型解释变异 } \\
R^{2}(\%)\end{array}$ & $\begin{array}{l}\text { 参考文献 } \\
\text { Reference }\end{array}$ \\
\hline RTRS & $A X \_109983408$ & $7 \mathrm{~B}$ & 23.3 & G & $3.50 \mathrm{E}-04$ & 10.5 & \\
\hline RTRV & $A X \_110003555$ & $1 \mathrm{~A}$ & 486.7 & $\mathrm{~T}$ & $5.71 \mathrm{E}-04$ & 9.6 & \\
\hline RTRV & $A X \_110543784$ & $1 \mathrm{~A}$ & 496.9 & A & $3.80 \mathrm{E}-06$ & 17.9 & \\
\hline RTRV & $A X \_109973556$ & $1 \mathrm{~A}$ & 520.9 & $\mathrm{C}$ & $3.38 \mathrm{E}-06$ & 16.9 & \\
\hline RTRV & $A X \_110111289$ & 1B & 384.3 & G & $3.01 \mathrm{E}-06$ & 17.0 & \\
\hline RTRV & $A X \_110492902$ & $1 \mathrm{D}$ & 382.2 & $\mathrm{~T}$ & $2.82 \mathrm{E}-05$ & 16.7 & \\
\hline RTRV & $A X \_108774755$ & $2 \mathrm{~A}$ & 125.9 & G & $2.48 \mathrm{E}-06$ & 17.4 & \\
\hline RTRV & $A X \_110536069$ & $2 \mathrm{~B}$ & 26.1 & $\mathrm{C}$ & $7.23 \mathrm{E}-04$ & 9.3 & \\
\hline RTRV & $A X \_109974238$ & $2 \mathrm{~B}$ & 785.8 & $\mathrm{~T}$ & $8.56 \mathrm{E}-04$ & 9.6 & \\
\hline RTRV & $A X_{-} 109586550$ & $2 \mathrm{D}$ & 632.0 & G & $6.33 \mathrm{E}-04$ & 9.5 & \\
\hline RTRV & $A X \_109933410$ & $3 \mathrm{~A}$ & 22.6 & G & 8.64E-04 & 7.2 & \\
\hline RTRV & $A X \_110935379$ & $3 \mathrm{~A}$ & 491.9 & $\mathrm{~T}$ & $1.29 \mathrm{E}-05$ & 14.9 & \\
\hline RTRV & $A X \_109931146$ & $3 \mathrm{~B}$ & 117.6 & $\mathrm{~T}$ & $8.08 \mathrm{E}-04$ & 9.4 & \\
\hline RTRV & $A X \_110075829$ & $3 \mathrm{~B}$ & 250.6 & G & $2.85 \mathrm{E}-06$ & 17.0 & \\
\hline RTRV & $A X \_110612422$ & $3 \mathrm{~B}$ & 403.5 & G & $3.16 \mathrm{E}-06$ & 16.9 & Shen et al. ${ }^{[30]}$ \\
\hline RTRV & $A X \_110411187$ & $3 \mathrm{~B}$ & 410.1 & G & $6.65 \mathrm{E}-05$ & 12.6 & \\
\hline RTRV & $A X \_110388860$ & $3 \mathrm{~B}$ & 466.1 & G & $2.83 \mathrm{E}-06$ & 17.1 & \\
\hline RTRV & $A X \_108977570$ & $3 \mathrm{~B}$ & 780.4 & $\mathrm{G}$ & $2.61 \mathrm{E}-06$ & 17.2 & Shen et al. ${ }^{[30]}$ \\
\hline RTRV & $A X \_110531944$ & $3 \mathrm{~B}$ & 780.4 & $\mathrm{~T}$ & $9.04 \mathrm{E}-04$ & 9.0 & \\
\hline RTRV & $A X \_109827784$ & $3 \mathrm{~B}$ & 780.7 & G & $8.90 \mathrm{E}-04$ & 9.0 & \\
\hline RTRV & $A X \_109279719$ & $3 \mathrm{~B}$ & 780.8 & G & 8.87E-04 & 9.0 & \\
\hline RTRV & $A X \_108848657$ & $3 \mathrm{~B}$ & 781.3 & $\mathrm{~T}$ & $4.03 \mathrm{E}-04$ & 10.2 & \\
\hline RTRV & $A X \_108783900$ & $3 \mathrm{~B}$ & 781.5 & G & $3.93 \mathrm{E}-04$ & 10.1 & \\
\hline RTRV & $A X \_109892051$ & $3 \mathrm{~B}$ & 821.0 & $\mathrm{~T}$ & $1.54 \mathrm{E}-04$ & 12.0 & \\
\hline RTRV & $A X \_109915316$ & $4 \mathrm{~B}$ & 554.8 & G & $3.27 \mathrm{E}-06$ & 16.8 & Ren et al. ${ }^{[29]}$ \\
\hline RTRV & $A X \_109364182$ & $4 \mathrm{~B}$ & 655.2 & $\mathrm{~T}$ & $8.98 \mathrm{E}-05$ & 13.2 & Ren et al. ${ }^{[29]}$ \\
\hline RTRV & $A X \_110522770$ & $5 \mathrm{~A}$ & 8.2 & $\mathrm{~T}$ & $2.32 \mathrm{E}-06$ & 17.5 & \\
\hline RTRV & $A X \_110044622$ & $5 \mathrm{~B}$ & 59.2 & $\mathrm{~T}$ & $2.11 \mathrm{E}-06$ & 17.5 & Ren et al. ${ }^{[29]}$ \\
\hline RTRV & $A X \_110366842$ & $5 \mathrm{~B}$ & 60.2 & G & $3.18 \mathrm{E}-06$ & 16.9 & \\
\hline RTRV & $A X \_109440517$ & $5 \mathrm{~B}$ & 504.0 & $\mathrm{~T}$ & $2.51 \mathrm{E}-06$ & 17.2 & \\
\hline RTRV & $A X \_108813077$ & $5 \mathrm{~B}$ & 517.9 & $\mathrm{C}$ & $2.88 \mathrm{E}-06$ & 17.0 & \\
\hline RTRV & $A X \_110045465$ & $5 \mathrm{~B}$ & 707.1 & $\mathrm{C}$ & $2.21 \mathrm{E}-05$ & 14.1 & Ren et al. ${ }^{[29]}$ \\
\hline RTRV & $A X \_108882877$ & $6 \mathrm{~A}$ & 557.9 & G & $5.68 \mathrm{E}-04$ & 9.9 & Shen et al. ${ }^{[30]}$ \\
\hline RTRV & $A X \_108740494$ & $6 \mathrm{~A}$ & 564.9 & G & $3.75 \mathrm{E}-06$ & 16.8 & \\
\hline RTRV & $A X \_110504351$ & $6 \mathrm{~A}$ & 596.0 & G & $8.66 \mathrm{E}-05$ & 12.7 & \\
\hline RTRV & $A X \_109907271$ & $6 \mathrm{~B}$ & 54.4 & $\mathrm{~T}$ & $3.12 \mathrm{E}-06$ & 17.0 & \\
\hline RTRV & $A X \_109580957$ & $6 \mathrm{~B}$ & 149.9 & $\mathrm{~T}$ & $1.18 \mathrm{E}-06$ & 19.6 & \\
\hline RTRV & $A X \_109308595$ & $6 \mathrm{~B}$ & 485.5 & $\mathrm{~T}$ & $1.34 \mathrm{E}-05$ & 15.2 & Ren et al. ${ }^{[29]}$ \\
\hline RTRV & $A X \_109364747$ & $7 \mathrm{~A}$ & 136.3 & $\mathrm{~T}$ & $7.92 \mathrm{E}-05$ & 12.3 & \\
\hline RTRV & $A X \_109024988$ & $7 \mathrm{~A}$ & 689.3 & $\mathrm{~T}$ & $9.92 \mathrm{E}-04$ & 8.9 & \\
\hline RTRV & $A X \_110444174$ & $7 \mathrm{~A}$ & 722.6 & $\mathrm{~T}$ & 3.97E-04 & 10.2 & \\
\hline RTRV & $A X \_109983408$ & $7 \mathrm{~B}$ & 23.3 & G & $3.46 \mathrm{E}-06$ & 17.3 & \\
\hline
\end{tabular}

RNRT: 相对根尖数; RARD: 相对根平均直径; RTRL：相对总根长; RTRS：相对总根表面积; RTRV：相对总根体积。

RNRT: relative number of root tips; RARD: relative average root diameter; RTRL: relative total root length; RTRS: relative total root surface area; RTRV: relative total root volume. 

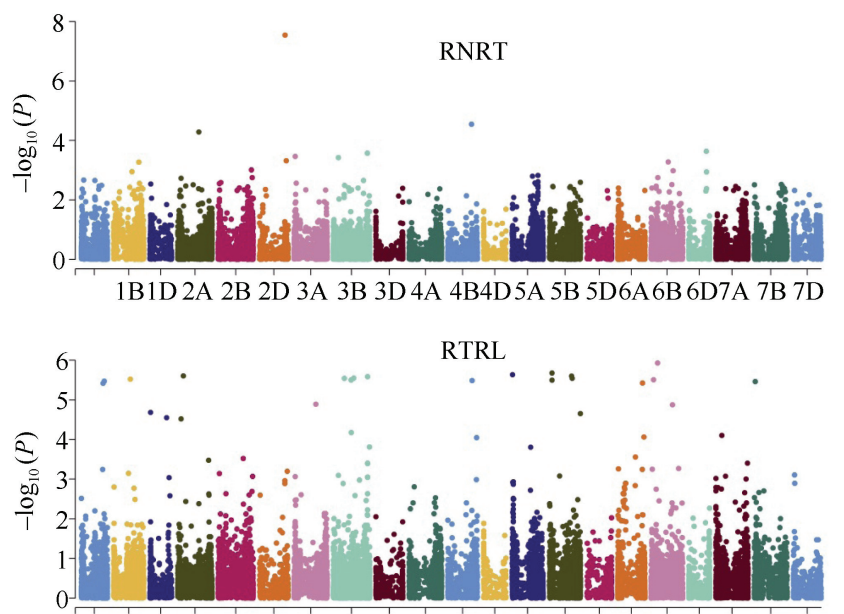

1B1D 2A 2B 2D 3A 3B 3D 4A 4B4D5A 5B 5D6A6B 6D7A 7B 7D

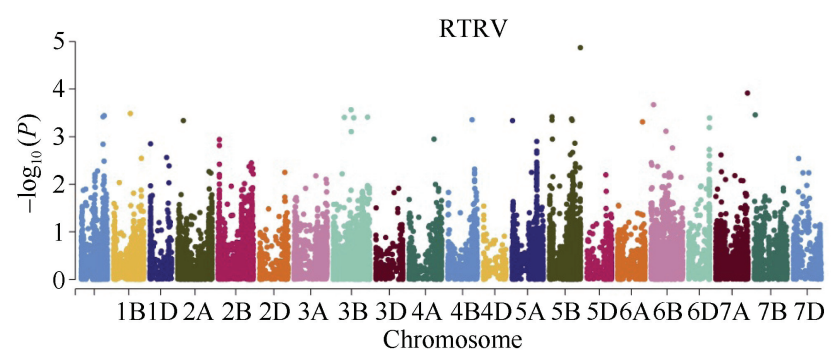

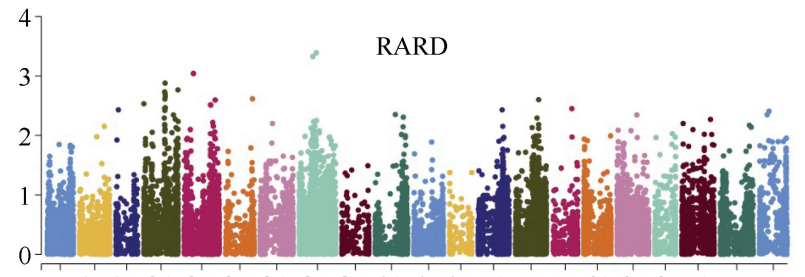

1B1D 2A 2B 2D 3A 3B 3D 4A 4B4D5A 5B 5D6A6B 6D7A 7B 7D RTRS

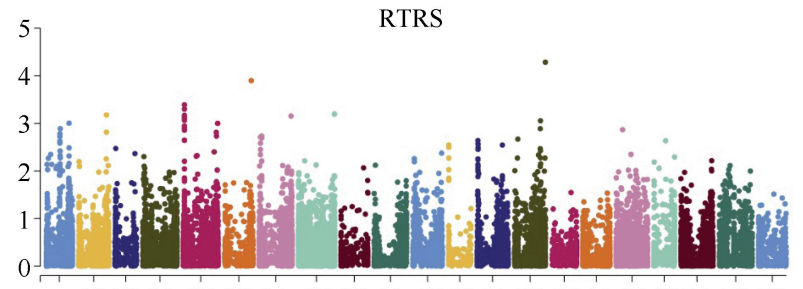

1B1D 2A 2B 2D 3A 3B 3D 4A 4B4D5A 5B 5D6A6B 6D7A 7B 7D Chromosome

图 3160 份普通小麦品系不同氮素条件下根系相关性状相对值全基因组关联分析曼哈顿图

Fig. 3 Manhattan plots for relative root system architecture (RSA) related traits under different nitrogen level in 160 common wheat lines

RNRT: 相对根尖数; RTRL：相对总根长; RTRS：相对总根表面积; RTRV：相对总根体积; RARD：相对根平均直径。

RNRT: relative number of root tips; RTRL: relative total root length; RTRS: relative total root surface area; RTRV: relative total root volume; RARD: relative average root diameter.

\section{3 讨论}

氮素是植物叶绿素、蛋白质、核酸、酶类、生 物碱、植物激素以及维生素类等物质的重要组成成 分, 是决定作物产量的首要因素。选育高氮素利用 效率品种是维持我国小麦生产安全的重要基础。长 期以来, 由于氮素利用效率评价困难, 育种家主要 依据地上部表型性状等来判断品种氮素利用效率, 对地下根系的作用认识不足，一定程度上限制了小 麦高产育种的进展 ${ }^{[3,6]}$ 。近年来, 小麦基因芯片的开 发和应用, 显著促进了数量性状遗传解析的进程 ${ }^{[9,26]}$ 。 本研究利用小麦 $660 \mathrm{~K}$ SNP 芯片, 在不同的氮素水 平下，尝试通过 GWAS 对小麦苗期不同环境下的根 系性状进行遗传解析, 以期发掘氮素利用效率相关 重要标记和遗传位点, 预测候选基因, 为通过分子 育种技术改良小麦氮素利用效率奠定基础。

本研究采用的小麦品系来源广泛, 包括地方品 种、高代品系和现代育成种。针对 3 种不同来源的 小麦材料，对其不同氮素水平条件下的根系相关性
状进行分析。多重比较结果表明, 高代品系和现代 育成种在不同氮素水平下根系构成相关性状均显著 高于地方品种, 表明人工选择对于小麦根系构型有 较大提升。小麦根系构型对于产量具有重要影响。 在人工选育过程中, 育种家在聚合高产基因, 选择 高产材料的同时 ${ }^{[27]}$, 一定程度上间接完成了小麦根 系构型的选择。然而, 针对不同氮素条件下根系构 型相关性状比值进行分析, 发现不同来源材料间存 在差异, 但并不显著。以上结果表明育种家在高产 育种过程中可以间接完成根系相关性状的选择, 但 是并不能有效开展氮素利用效率相关性状的选择, 因此, 通过开展不同氮素环境下根系表型鉴定, 间 接发掘氮素利用效率相关位点, 对于促进小麦高氮 素利用效率选择和育种是十分必要的。

基因多态性是保证关联分析结果高效、准确的 前提。本研究采用 160 份品系的基因多态性为 0.34 , 与之前报道接近 ${ }^{[28]}$, 可基本反映黄淮和北部冬麦区 的品种多样性。相较于 $\mathrm{D}$ 基因组, A 和 $\mathrm{B}$ 基因组具 
有较高的遗传多态性, 这与前人研究结果一致 ${ }^{[9]}$ 。本 研究采用品系遗传变异丰富, 适用于 GWAS 分析。 PCA 分析表明人工选择对群体结构分层具有显著影 响。群体结构可导致关联分析结果出现假阳性 ${ }^{[28]}$ 。 因此, 采用结合群体结构 (PCA)和亲缘关系(K)的混 合线性模型(MLM)进行 GWAS, 以避免假阳性的出 现。LD 衰减是影响关联分析准确度的重要因素, 不 同自然群体的 LD 衰减距离不同。一般来说, 普通小 麦 LD 衰减距离在 $1.5 \sim 15.0 \mathrm{cM}$ 范围内 ${ }^{[9,26]}$ 。本研究 中, 全基因组 $\mathrm{LD}$ 平均衰减距离为 $8 \mathrm{Mb}$ 。全基因组 的平均标记间距(0.097)远低于 LD 衰减距离, 标记 密度可满足关联分析要求 ${ }^{[21]}$ 。

相较于双亲群体, 自然群体积累了广泛的重组 事件，提供了更多有效重组信息，可在短时间内发 现更多关联位点。除此之外, GWAS 分辨率较高, 有 利于遗传位点的进一步精细定位和图位克隆。本研 究共计检测到 34 个与氮素利用效率显著关联的位 点, 在 21 条染色体上均有分布。其中, 与 RNRT 关 联的显著位点 $A X \_109931146(3 \mathrm{~B}, 117.6 \mathrm{Mb})$, 与 RTRS 关联的显著位点 $A X \_110522770(5 \mathrm{~A}, 8.2 \mathrm{Mb})$ 、 $A X \_109907271(6 \mathrm{~B}, 54.4 \mathrm{Mb})$, 与 RTRV 关联的显著 位点 $A X \_110612422(3 \mathrm{~B}, 403.5 \mathrm{Mb}) 、 A X \_110531944$ (3B, 780.4Mb), $A X \_109915316(4 \mathrm{~B}, 554.8 \mathrm{Mb}) 、$ $A X \_109364182(4 \mathrm{~B}, 655.2 \mathrm{Mb}) 、 A X_{-} 110044622(5 \mathrm{~B}$, $59.2 \mathrm{Mb}) 、 A X \_110045465(5 \mathrm{~B}, 707.1 \mathrm{Mb}) 、$ $A X \_108882877(6 \mathrm{~A}, 557.9 \mathrm{Mb})$ 和 $A X \_109308595$ $(6 \mathrm{~B} 、 485.5 \mathrm{Mb})$ 与 Ren 等 ${ }^{[29]}$ 和沈兴 ${ }^{[30]}$ 发掘到的多个 氮素利用效率相关位点重叠或一致。这些位点存在 氮素利用效率相关基因的可能性较大，但迄今尚末 克隆, 无法探讨其遗传调控机制。另外, 在这些区域, 我们发现了部分生长素响应(auxin responsive gene, $\mathrm{ARF}$ )相关基因，但由于置信区间较大，仍不可确定 为候选基因, 下一步将针对这些区间开展精细定位 工作。除此之外，前人针对小麦氮素利用效率遗传 机制也开展了多项研究，在小麦 $2 \mathrm{~A} 、 2 \mathrm{~B} 、 4 \mathrm{~A} 、 5 \mathrm{~A}$ 、 $7 \mathrm{~A}$ 和 7B 染色体上发现一批控制小麦氮素利用效率 相关遗传位点 ${ }^{[16-19]}$, 但是由于多数研究采用的标记
为传统的 RFLP、SSR 或者 DArT 标记, 根据现有小 麦遗传或物理图谱, 无法进行可靠的对比, 暂时无 法确定是否与本研究发现位点一致。

基于 NCBI 数据库, 利用与氮素利用效率相关 性状显著关联的 SNP 标记的侧翼序列通过 BLASTn 发掘候选基因, 在 3B 染色体( $A X_{-}$109827784)上检测 到一个编码 E3-泛素蛋白连接酶的候选基因。E3-泛 素蛋白连接酶是一种多类型的蛋白家族, 在植物氮 素吸收、根系和幼芽生长发育过程中起着十分重要 的作用 ${ }^{[31-32]}$ 。虽然基于关联标记的生物信息分析是发 掘复杂农艺性状相关候选基因的有效方法, 然而, 由 于小麦根系构型建成涉及到多个复杂的生理、生化过 程, 后续工作将围绕候选基因的作用进行验证。

氮素利用效率是一个十分复杂的农艺性状, 相 关研究较为滞后, 严重制约了高产、广适品种的培 育。由于氮素利用效率在田间育种时无法及时评价 表型, 基于分子标记的 MAS 育种是提升品种氮素利 用效率最为重要的途径之一。因此, 研究品种间氮 素利用效率差异, 篮选高氮素利用效率品种, 对培 育新的优良品种具有重要价值。本研究共计发现 34 个氮素利用效率关联位点。其中, 存在多个位点与 多个性状关联, 如 $1 \mathrm{~A}$ 染色体 $486.7 \mathrm{Mb} 496.9 \mathrm{Mb}$ 处 与 RTRS 和 RTRV 均显著关联, $1 \mathrm{~A}$ 染色体 $515.8 \mathrm{Mb}$ $520.9 \mathrm{Mb}$ 处与 RTRL、RTRS 和 RTRV 均显著关联、 $2 B$ 染色体 $25.5 \mathrm{Mb} 26.1 \mathrm{Mb}$ 处与 RTRL 和 RTRV 等 性状显著关联, 这些位点相对更为可靠, 可进一步 转化为 $\mathrm{KASP}^{[33-34]}$ 或 $\mathrm{STARP}^{[35]}$ 标记, 用于分子标记 辅助育种和 QTL 精细定位。在所有实验材料中, 徐 麦 856、郑麦 366、济南 4 号、临麦 6 号、兰考 358 、 山农 587、济麦 52、山农 0431、山农 22、济麦 37 、 鲁原 502、山农 1565 、汶农 14、鲁麦 20、金麦 296 等品种具有较高的氮素利用效率, 农艺性状优良, 含有较多优异等位基因(15 21 个), 可作为亲本, 用以导 入或聚合优异等位基因, 提升育种材料的氮素利用 效率(表 4)。基于以上结果, 下一步研究工作将主要 集中于关联位点的验证和育种家友好型 KASP 标记 的开发, 为提升氮素利用效率提供参考。

表 4 高氮素利用效率小麦品种

Table 4 Wheat accessions with higher nitrogen use efficiency

\begin{tabular}{|c|c|c|c|c|c|c|}
\hline \multirow{2}{*}{$\begin{array}{c}\text { 品种 } \\
\text { Cultivar }\end{array}$} & \multicolumn{5}{|c|}{ 性状 Trait } & \multirow{2}{*}{$\begin{array}{c}\text { 优异等位基因数 } \\
\text { Number of favorable alleles }\end{array}$} \\
\hline & RTRL & RTRS & RARD & RTRV & RNRT & \\
\hline 徐麦 856 Xumai 856 & 2.356 & 1.149 & 0.748 & 1.415 & 4.850 & 17 \\
\hline 郑麦 366 Zhengmai 366 & 2.474 & 1.489 & 0.741 & 2.085 & 5.747 & 18 \\
\hline
\end{tabular}




\begin{tabular}{|c|c|c|c|c|c|c|}
\hline \multirow{2}{*}{$\begin{array}{c}\text { 品种 } \\
\text { Cultivar }\end{array}$} & \multicolumn{5}{|c|}{ 性状 Trait } & \multirow{2}{*}{$\begin{array}{c}\text { 优异等位基因数 } \\
\text { Number of favorable alleles }\end{array}$} \\
\hline & RTRL & RTRS & RARD & RTRV & RNRT & \\
\hline 济南 4 号 Jinan 4 & 2.495 & 1.238 & 0.722 & 0.985 & 3.951 & 16 \\
\hline 临麦 6 号 Linmai 6 & 2.548 & 1.177 & 0.584 & 1.870 & 8.476 & 15 \\
\hline 兰考 358 Lankao 358 & 2.630 & 1.336 & 0.651 & 1.707 & 4.918 & 18 \\
\hline 山农 587 Shannong 587 & 2.674 & 1.401 & 0.678 & 0.903 & 6.599 & 15 \\
\hline 济麦 52 Jimai 52 & 2.687 & 1.433 & 0.531 & 1.988 & 11.925 & 16 \\
\hline 山农 0431 Shannong 0431 & 2.856 & 1.600 & 0.526 & 1.280 & 6.991 & 17 \\
\hline 山农 22 Shannong 22 & 2.884 & 1.206 & 0.690 & 1.554 & 5.695 & 19 \\
\hline 济麦 37 Jimai 37 & 2.907 & 1.385 & 0.587 & 0.968 & 3.233 & 20 \\
\hline 鲁原 502 Luyuan 502 & 3.114 & 1.520 & 0.729 & 2.882 & 15.500 & 15 \\
\hline 山农 1565 Shannong 1565 & 3.230 & 1.286 & 0.466 & 1.599 & 8.706 & 16 \\
\hline 汶农 14 Wennong 14 & 3.648 & 1.254 & 0.507 & 0.918 & 6.894 & 17 \\
\hline 鲁麦 20 Lumai 20 & 4.029 & 2.079 & 0.432 & 1.534 & 15.089 & 21 \\
\hline 金鉴麦 296 Xinmai 296 & 5.828 & 2.164 & 0.481 & 2.113 & 24.547 & 20 \\
\hline
\end{tabular}

RTRL: 相对总根长; RTRS: 相对总根表面积; RARD: 相对根平均直径; RTRV: 相对总根体积; RNRT: 相对根尖数。

RTRL: relative total root length; RTRS: relative total root surface area; RARD: relative average root diameter; RTRV: relative total root volume; RNRT: relative number of root tips.

\section{4 结论}

本研究利用 160 份小麦品系, 通过测定苗期根 系构型相关性状，间接开展氮素利用效率相关性状 的 GWAS 分析，共计检测到 34 个关联位点。发掘到 的遗传位点及其关联标记可开发为 KASP 标记，用 于篮选高氮素利用效率小麦品种, 篮选出的品种, 可作为亲本材料应用于小麦高产育种中。

附图和附表 请见网络版: 1) 本刊网站 http://zwxb. chinacrops.org/; 2) 中国知网 http://www.cnki.net/; 3) 万方数据 http://c.wanfangdata.com.cn/Periodicalzuowxb.aspx。

\section{References}

[1] Chen J G, Zhang Y, Tan Y W, Zhang M, Zhu L L, Xu G H, Fan X $\mathrm{R}$. Agronomic nitrogen-use efficiency of rice can be increased by driving OsNRT2.1 expression with the OsNAR2.1 promoter. Plant Biotechnol J, 2016, 14: 1705-1715.

[2] Sandrine R, Alain G, Laurence L. Signal interactions in the regulation of root nitrate uptake. $J$ Exp Bot, 2014, 65: 5509-5517.

[3] Maccaferri M, El-Feki W, Nazemi G, Salvi S, Canè M A, Colalongo M C, Stefanelli S, Tuberosa R. Prioritizing quantitative trait loci for root system architecture in tetraploid wheat. $J$ Exp Bot, 2016, 67: 1161-1178.

[4] Kabir M R, Liu G, Guan P F, Wang F, Khan A A, Ni Z F, Yao Y Y, Hu Z R, Xin M M, Peng H R. Mapping QTLs associated with root traits using two different populations in wheat (Triticum aestivum L.). Euphytica, 2015, 206: 175-190.

[5] de Dorlodot S, Forster B, Pagès L, Price A, Tuberosa R, Draye X.
Root system architecture: opportunities and constraints for genetic improvement of crops. Trends Plant Sci, 2007, 12: 474-481.

[6] Bishopp A, Lynch J P. The hidden half of crop yields. Nat Plants, 2015, 1:15117.

[7] Paez-Garcia A, Motes C M, Scheible W R, Chen R, Blancaflor E $\mathrm{B}$, Monteros M J. Root traits and phenotyping strategies for plant improvement. Plants, 2015, 4: 334-355.

[8] Osmont K S, Sibout R, Hardtke C S. Hidden branches: developments in root system architecture. Annu Rev Plant Biol, 2007, 58: 93-113.

[9] Liu J D, He Z H, Rasheed A, Wen W E, Yan J, Zhang P Z, Wan Y $\mathrm{X}$, Zhang Y, Xie C J, Xia X C. Genome-wide association mapping of black point reaction in common wheat (Triticum aestivum L.). BMC Plant Biol, 2017, 17: 220.

[10] Dong Y, Liu J D, Zhang Y, Geng H W, Awais R, Xiao Y G, Cao S H, Fu L P, Yan J, Wen WW, Zhang Y, Jing R L, Xia X C, He Z H. Genome-wide association of stem water soluble carbohydrates in bread wheat. PLoS One, 2016, 11: e0164293.

[11] Rasheed A, Xia X C, Ogbonnaya F, Mahmood T, Zhang Z, Mujeeb-Kazi A M, He Z H. Genome-wide association for grain morphology in synthetic hexaploid wheats using digital imaging analysis. BMC Plant Biol, 2014, 14: 12.

[12] Guo J, Shi W P, Zhang Z, Cheng J Y, Sun D Z, Yu J, Li X L, Guo $\mathrm{P}$ Y, Hao C Y. Association of yield-related traits in founder genotypes and derivatives of common wheat (Triticum aestivum L.). BMC Plant Biol, 2018, 18: 387.

[13] Maccaferri M, Ricci A, Salvi S, Milner S G, Noli E, Martelli P L, Casadio R, Akhunov E, Scalabrin S, Vendramin V, Ammar K, Blanco A, Desiderio F, Distelfeld A, Dubcovsky J, Fahima T, Faris J, Korol A, Massi A, Mastrangelo A M, Morgante M, Pozniak C, N'Diaye A, Xu S, Tuberosa R. A high-density, SNP-based consensus map of tetraploid wheat as a bridge to 
integrate durum and bread wheat genomics and breeding. Plant Biotechnol J, 2015, 13: 648-663.

[14] Bulli P, Zhang J, Chao S, Chen X, Pumphrey M. Genetic architecture of resistance to stripe rust in a global winter wheat germplasm collection. G3: Genes Genom Genet, 2016, 8: $2237-$ 2253.

[15] Lian X M, Xing Y Z, Yan H, Xu C G, Li XH, Zhang Q F. QTL for low nitrogen tolerance at seedling stage identified using a recombinant inbred line population derived from an elite rice hybrid. Theor Appl Genet, 2005, 112: 85-96.

[16] An D G, Su J Y, Liu Q Y, Zhu Y G, Tong Y P, Li M J, Jing R L, Lin B, Li Z S. Mapping QTL for nitrogen uptake in relation to the early growth of wheat (Triticum aestivum L.). Plant Soil, 2006, 284: 73-84.

[17] Quarrie S A, Steed A, Calestani C, Semikhodskii A, Lebreton C, Chinoy C, Steele N, Pljevljakusić D, Waterman E, Weyen J, Schondelmaier J, Habash Z D, Farmer P, Saker L, Clarkson D T, Abugalieva A, Yessimbekova M, Turuspekov Y, Abugalieva S, Tuberosa R, Sanguineti M C, Hollington P A, Aragués R, Royo A, Dodig D. A high-density genetic map of hexaploid wheat (Triticum aestivum $\mathrm{L}$.) from the cross Chinese Spring $\times$ SQ1 and its use to compare QTLs from grain yield across a range of environments. Theor Appl Genet, 2005, 110: 865-880.

[18] Fontaine J X, Ravel C, Pageau K, Heumez E, Dubois F, Hirel B. A quantitative genetic study for elucidating the contribution of glutamine synthetase, glutamate dehydrogenase and other nitrogen-related physiological traits to the agronomic performance of common wheat. Theor Appl Genet, 2009, 119: 645-662.

[19] Cui F, Fan X, Zhao C, Zhang W, Chen M, Ji J, Li J. A novel genetic map of wheat: utility for mapping QTL for yield under different nitrogen treatments. BMC Genet, 2014, 15: 57.

[20] Hoagland D R, Arnon D I. The water-culture method for growing plants without soil. California Agric Exp Station Circular, 1950, 347: 1-32.

[21] Saghai-Maroof M A, Soliman K M, Jorgensen R A, Allard R W L. Ribosomal DNA spacer-length polymorphisms in barley: Mendelian inheritance, chromosomal location, and population dynamics. Proc Natl Acad Sci USA, 1984, 81: 8014-8018.

[22] Breseghello F, Sorrells M E. Association mapping of kernel size and milling quality in wheat (Triticum aestivum L.) cultivars. Genetics, 2006, 172: 1165-1177.

[23] Kump K L, Bradbury P J, Wisser R J, Buckler E S, Belcher A R, Oropeza-Rosas M A, Balint-Kurti P J. Genome-wide association study of quantitative resistance to southern leaf blight in the maize nested association mapping population. Nat Genet, 2011, 43: 163.
[24] Zhu C, Gore M, Buckler E S, Yu J. Status Prospects of association mapping in plants. Plant Genome, 2008, 1: 5-20.

[25] Poland J A, Bradbury P J, Buckler E S, Nelson R. Genome-wide nested association mapping of quantitative resistance to northern leaf blight in maize. Proc Natl Acad Sci USA, 2011, 108: 6893-6898.

[26] Hao C Y, Wang Y Q, Chao S M, Li T, Liu H X, Wang L F, Zhang $X$ Y. The iSelect 9K SNP analysis revealed polyploidization induced revolutionary changes and intense human selection causing strong haplotype blocks in wheat. Sci Rep (UK), 2017, 4: 41247.

[27] Wang Y M, Hou J, Liu H, Li T, Wang K, Hao C Y, Liu H, Zhang $\mathrm{X}$ Y. TaBT1, affecting starch synthesis and thousand kernel weight, underwent strong selection during wheat improvement. $J$ Exp Bot, 2019, 70: 1497-511.

[28] Chen X J, Min D H, Tauqeer A Y, Hu Y G. Genetic diversity, population structure and linkage disequilibrium in elite Chinese winter wheat investigated with SSR markers. PLoS One, 2012, 7: e44510.

[29] Ren D, Fang X, Jiang P, Zhang G, Hu J, Wang X, Wang H. Genetic architecture of nitrogen-deficiency tolerance in wheat seedlings based on a nested association mapping (NAM) population. Front Plant Sci, 2018, 9: 845.

[30] 沈兴. 小麦氮营养特性的基因型差异及其遗传解析. 山东农 业大学硕士学位论文, 山东泰安, 2018. pp 28-44.

Shen X. Genotypic Differences and Genetic Analysis in Nitrogen Nutritional Characteristics of Wheat. MS Thesis of Shandong Agricultural University, Tai'an, Shandong, China, 2018. pp 28-44 (in Chinese with English abstract).

[31] Craig A, Ewan R, Mesmar J, Gudipati V, Sadanandom A. E3 ubiquitin ligases and plant innate immunity. $J$ Exp Bot, 2009, 60: 1123-1132.

[32] Park G G, Park J J, Yoon J M, Yu S N. An GH A RING finger E3 ligase gene, Oryza sativa Delayed Seed Germination 1 (OsDSG1), controls seed germination and stress responses in rice. Plant Mol Biol, 2010, 74: 467-478.

[33] Semagn K, Babu R, Hearne S, Olsen M. Single nucleotide polymorphism genotyping using Kompetitive Allele Specific PCR (KASP): overview of the technology and its application in crop improvement. Mol Breed, 2014, 33: 1-14.

[34] Rasheed A, Hao Y F, Xia X C, Khan A, Xu Y B, Varshney R K, $\mathrm{He} \mathrm{Z} \mathrm{H}$. Crop breeding chips and genotyping platforms: progress, challenges, and perspectives. Mol Plant, 2017, 10: 1047-1064.

[35] Long Y M, Chao W S, Ma G J, Xu S S, Qi L L. An innovative SNP genotyping method adapting to multiple platforms and throughputs. Theor Appl Genet, 2016, 130: 597-607. 